HUB-EP-97/03

hep-th/9701053

January 1997

\title{
Classical and quantum aspects of 4 dimensional black holes*
}

\author{
Klaus Behrndt ${ }^{\mathrm{a}}$ \\ anstitut für Physik, Humboldt Universität, \\ Invalidenstraße 110, 10115 Berlin, Germany
}

In this talk I address some aspects in the recent developments for $N=2$ black holes in 4 dimensions. I restrict myself on axion-free solutions that can classically be related to intersections of isotropic $D$ - or $M$-branes. After reviewing of some classical properties I include corrections coming from a general cubic prepotential. On the heterotic side these are quantum corrections for these black hole solutions. Finally, I discuss a microscopic interpretation of the entropy for the extremal as well as near-extremal black hole.

\section{Introduction}

This paper is based on talks that I have given at the Buckow meeting as well as two further occasions. The development was so rapid in recent times, that I could not resist to add some further points that have not been discussed in Buckow. I hope the reader will get a more complete picture of this interesting topic.

In the last year a complete new picture of 4dimensional (4d) black holes appeared. The main ingredient came from the $D$-branes [1] and the discovery that they are black $p$-brane solutions of the RR sector of string theory [2]. Soon, it has been shown that the $4 \mathrm{~d}$ black holes can be seen as intersections or bound states of these $D$-branes. The interesting point is, that it provides a microscopic picture of the black holes in term of open string states. Thus, one can give the BekensteinHawking entropy a statistical interpretation. The Hawking radiation appears as a recombination of two open strings to a departing closed string. In this picture seems to be no room for an information paradox.

*Contribution for the "30th Ahrenshoop International Symposium on the Theory of Elementary Particles", Buckow, Germany, 27-31 Aug 1996
In the first part I will introduce the basic properties of supersymmetric $4 \mathrm{~d}$ black holes and relate them to intersections of $D$-branes. In the second part I am going to discuss corrections coming from a general cubic prepotential. On the heterotic side these are quantum corrections and on the type II side these corrections are related to the Calabi-Yau (CY) compactification. Finally, using the microscopic picture I describe the excitation and radiation of the black hole and argue that the Bekenstein-Hawking entropy coincides with statistical entropy, which counts the degeneracy of states.

\section{4d susy black holes}

Static supersymmetric black holes in 4 dimensions can be classified in 4 groups. Keeping only one gauge field and one scalar fields these black holes are solutions of the effective action

$S=\int d^{4} x \sqrt{|g|}\left\{R-2(\partial \phi)^{2}+e^{-2 a \phi} F^{2}\right\}$

and are classified by the scalar coupling $a$. Generally, one can find solutions for all $a$ [3] but supersymmetry allows only the discrete values $a=0,1 / \sqrt{3}, 1, \sqrt{3}$. The metric is given by

$d s^{2}=-H^{-\frac{2}{1+a^{2}}} d t^{2}+H^{\frac{2}{1+a^{2}}} d \vec{x}^{2}$ 


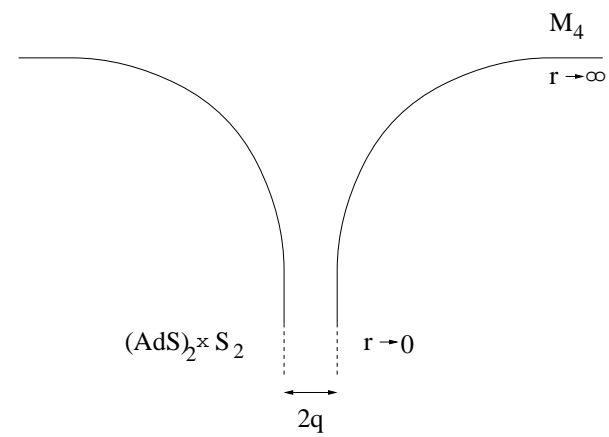

Figure 1. The two asymptotic limits of the Reissner-Nordstrøm solution; flat space time at infinity and in the throat region $(r \rightarrow 0)$ the Bertotti-Robertson space.

where $H$ is a harmonic function. For $a=0$ we have the standard selfdual Reissner-Nordstrøm solution and for $a=1$ it is the dilaton black hole hole. For $a=1 / \sqrt{3}$ or $a=\sqrt{3}$ one can get this black hole solution from the compactification of the 5d Reissner-Nordstrøm resp. as Kaluza-Klein black hole. Let us look in more detail on the case $a=0$. This solution can be written as

$d s^{2}=-\frac{1}{\left(1+\frac{q}{r}\right)^{2}} d t^{2}+\left(1+\frac{q}{r}\right)^{2}\left[d r^{2}+r^{2} d \Omega\right]$

and for $r \rightarrow 0$ and $r=q e^{\eta / q}$ this solution becomes

$d s^{2}=-e^{2 \eta / q} d t^{2}+d \eta^{2}+q^{2} d \Omega$.

This is a Bertotti-Robertson space $\left(A d S_{2} \times S_{2}\right)$ which is non-singular (see figure 1).

Generically, the Reissner-Nordstrøm solution breaks $1 / 2$ of $N=2$ supersymmetries, but this asymptotic geometry restores all of them. Thus, this solution can be seen as a soliton that interpolates between two maximal supersymmetric vacua, at infinity and on the horizon. All other solutions with non-vanishing $a$ are singular at $r=0$, but allow more unbroken supersymmetries. The $a=\sqrt{3}$ solution e.g. breaks only $1 / 2$ of $N=2$ supersymmetries in $D=10$.
In order to get the corresponding non-extreme solution we have to replace [4]

$$
\begin{aligned}
& d t^{2} \rightarrow\left(1-\frac{\mu}{r}\right) d t^{2}, d r^{2} \rightarrow d r^{2} /\left(1-\frac{\mu}{r}\right) \\
& H=1+\frac{q}{r} \rightarrow 1+\frac{\mu \sinh ^{2} \alpha}{r}
\end{aligned}
$$

where $\mu$ is the non-extremality parameter and $\alpha$ is a boost parameter. The extreme limit is: $\mu \rightarrow 0$, $\alpha \rightarrow \infty$, but $\mu \sinh ^{2} \alpha=q$ (see also the last paragraph). For all non-vanishing $a$ the horizon at $r=\mu$ touches the singularity in the extreme case. The situation becomes even worse if we would include angular momentum, where the singularity is naked in the extreme limit.

\section{Black holes as intersections of branes}

All these solutions have been generalised to the case of more gauge fields and scalars. It is possible to see the $a=\sqrt{3}$ solution as a fundamental state and all others as bound state of this fundamental one [5]. In 10 dimensions this idea was confirmed by the interpretation of these solutions as intersections of $D$-branes, which are $p$ brane solutions of the RR sector of type II string theory. They are hypersurfaces on which endpoints of open strings are attached [2] and since the open strings are fixed in the transverse directions (=Dirichlet boundary conditions), these branes are called Dirichlet-branes (or $D$-branes). This was a major breakthrough in a new view, not only on these solutions but also on strings or domain walls in 4 dimensions [6].

Starting point was the reinterpretation of the Güven solutions [7] as intersections of the 2-brane solution of the $11 \mathrm{~d}$ supergravity ( $M$-2-brane) [8]. Soon after this paper appeared all other solutions have been reinterpreted (see e.g. [9 11]). Let us start with some comments about these brane solutions. First, they can have all dimensions, starting from the (-1)-instanton until the complete $10 \mathrm{~d}$ space, the 9-brane. The odd branes are solutions of type IIB and the even branes of IIA superstring theory. Furthermore, the (-1)-brane is defined in Euclidian space time and the 7 - \& 8-branes are not asymptotically flat (the 9-brane is the flat space time).

Assuming that $z_{i}$ are the world-volume coordinates and $x_{m}$ are the transversal directions these 


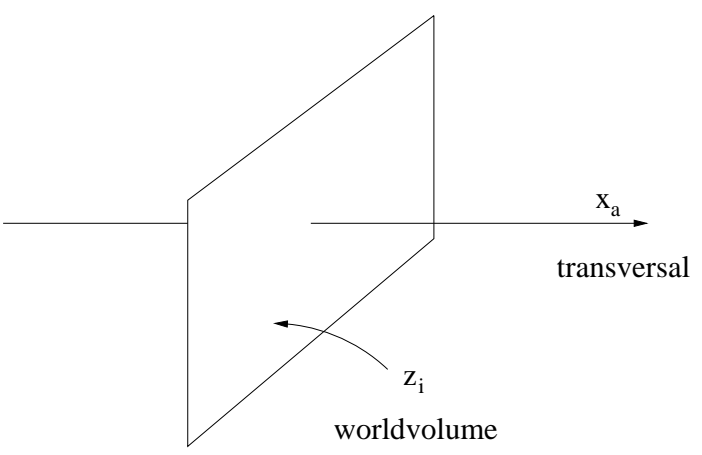

Figure 2. A single isotropic branes as given in (6).

branes can be written as (see figure 2)

$$
\begin{aligned}
& d s^{2}=\frac{1}{\sqrt{H}}\left(-d t^{2}+d z_{i}^{2}\right)+\sqrt{H} d x_{m}^{2} \\
& F=\left\{\begin{array}{l}
d H \wedge d t \wedge . . \wedge d z_{i}, p \leq 3 \\
* d H \wedge d t \wedge . . \wedge d z_{i}, p \geq 3
\end{array}\right. \\
& e^{-2 \phi} \sim H^{\frac{p-3}{2}}, \quad \partial^{2} H(x)=0 .
\end{aligned}
$$

These solutions are electric if $p<3$, magnetic if $p>3$ and for $p=3$ we have the self-dual 3brane. In addition we have of course the brane solutions of the NS sector, the fundamental string and the solitonic 5-brane. This completes the set of charged brane solutions of the 10d effective string theory. As neutral solutions we have the wave and the Taub-NUT soliton, which are pure gravitational solutions.

Since the type IIA theory follows by compactification of the $11 \mathrm{~d}$ supergravity, all even branes are compactified solutions too. The 0- \& 6-brane appear as KK solutions, whereas the 2 - \& 4-brane are compactified $M-2-\& M$-5-branes. Like the open string picture in $10 \mathrm{~d}$, we have in $11 \mathrm{~d}$ the possibility that an membrane can be open and end on a 5-brane 12. In difference, however, the open membrane has to end on a 5 -brane whereas the open string can also be free. The $M$-brane solutions of 11d supergravity are

$$
\begin{aligned}
& d s_{2}^{2}=H^{-\frac{2}{3}}\left(-d t^{2}+d z_{i}^{2}\right)+H^{\frac{1}{3}} d x_{m}^{2}, \\
& d s_{5}^{2}=H^{-\frac{1}{3}}\left(-d t^{2}+d z_{i}^{2}\right)+H^{\frac{2}{3}} d x_{m}^{2} .
\end{aligned}
$$

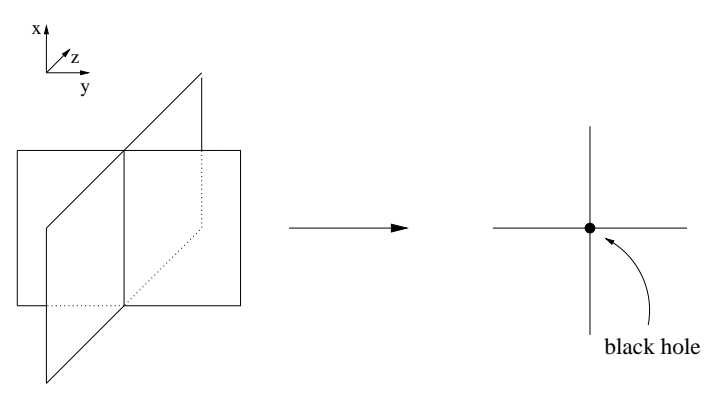

Figure 3. Two 3-branes intersect over a string. Here $x$ is the common worldvolume and $y$ and $z$ are relative transversal coordinates. If we intersect two such objects we get a black hole at the intersection.

It is still an open question what is the $11 \mathrm{~d}$ analogue of the $D$-8-branes solution in 10d.

So far we have discussed single branes which yield after compactification to $4 \mathrm{~d}$ the $a=\sqrt{3}$ black holes. In order to get also the other black hole solutions, we need to form bound states of branes. These are states, where two or more branes intersect each other perpendicularly (see figure 3 ). The construction rules for these configurations can be found in 9] or using $T$-duality in [10]. E.g., two $D$-3-branes, which intersect over a string (= common worldvolume) have the metric

$$
\begin{aligned}
& d s_{I I B}^{2}=\frac{1}{\sqrt{H_{1} H_{2}}} d u d v+\sqrt{H_{1} H_{2}} d x_{m}^{2} \\
& \quad+\sqrt{\frac{H_{1}}{H_{2}}}\left(d x_{6}^{2}+d x_{7}^{2}\right)+\sqrt{\frac{H_{2}}{H_{1}}}\left(d x_{8}^{2}+d x_{9}^{2}\right)
\end{aligned}
$$

where $u, v=z \pm t$ are the common world volume of the two 3-branes, $x_{m}$ are 4 transversal coordinates and $x_{6} . . x_{9}$ are the relative transversal coordinates (they are worldvolume of one brane but transversal for the other). Compactifying this configuration gives however a singular solution in 4 dimensions, $G_{z z}$ and $G_{55}$ are singular. To keep these compactification radii finite we can make a boost along $z$ and put a Taub-NUT soliton in the $4 \mathrm{~d}$ transversal space, i.e.

$$
\begin{aligned}
& d u d v \rightarrow d u d v+H_{0} d u^{2} \\
& d x_{m} d x_{m} \rightarrow \frac{1}{H_{3}}\left(d x_{5}+\vec{V} d \vec{x}\right)^{2}+H_{3} d \vec{x} d \vec{x},
\end{aligned}
$$


where: $\epsilon_{i j k} \partial_{j} V_{k}=\partial_{i} H_{3}$. This is a non-singular type IIB solution. An analog solution on the type IIA side can be uplifted to 11d. This solution is given by three 5 -branes which intersect over a string [9]

$$
\begin{aligned}
d s_{M}^{2} & =\left(H_{1} H_{2} H_{3}\right)^{-\frac{1}{3}}\left[d u d v+H_{0} d u^{2}\right. \\
& \left.+H_{1} H_{2} H_{3} d \vec{x}^{2}+H_{A} \omega^{A}\right]
\end{aligned}
$$

where $\omega^{A}$ are three $2 \mathrm{~d}$ line elements. The configuration with coinciding harmonic functions has been discussed before in [8]. These intersections contain all 4 classes of black hole upon compactification to $4 \mathrm{~d}$. The number of non-trivial harmonic functions determines the solution: the $a=0, \frac{1}{\sqrt{3}}, 1$ or $\sqrt{3}$ solution is given by an intersection with $4,3,2$ or 1 non-trivial harmonic functions (or branes).

These examples show also an interesting point concerning the singularity of the $4 \mathrm{~d}$ black holes. The $D$-3-brane as well as the $M$-branes are nonsingular objects 13. The compactification of (8) to $6 \mathrm{~d}$ gives the self-dual string. Also this object is non-singular - even behind the horizon is no singularity hidden, like for the Reissner-Nordstrøm black hole. A further compactification to $4 \mathrm{~d}$ gives us the singular $a=1$ black hole. Thus, the singularity of this black hole is a consequence of the compactification. The same is true for the other black holes. The $a=\sqrt{3}$ black hole is a compactified single brane. So, we can take either the $D$-3-branes, $M$-2- or the $M$-5-brane (7) and compactify them to $4 \mathrm{~d}$ and get the $a=\sqrt{3}$ black hole. Also for the $a=1 / \sqrt{3}$ black holes we can find intersections of non-singular branes. The magnetic case is given by the intersection (10) if we set $H_{0}=0$, i.e. an intersection of three $M$-5-branes. The electric solution corresponds to an intersection of three $M$-2-branes

$$
\begin{array}{r}
d s_{M}^{2}=\left(H_{1} H_{2} H_{3}\right)^{-\frac{2}{3}}\left[-d t^{2}+\right. \\
\left.+H_{1} H_{2} H_{3} d x_{m}+H_{A} \omega^{A}\right]
\end{array}
$$

where: $m=1 . .4$. Finally, the $a=0$ black hole can be obtained from the intersection $3 \times 3 \times 3 \times 3$ or $2 \times 2 \times 5 \times 5$. But also the 5 d black hole or black string, appearing after compactification over the $\omega^{A}$-part in (11) or (10), are non-singular. Thus, all black holes, that we had described in section 2 , can be seen as compactification of non-singular solutions. The singularities are a consequence of the compactification [14]. Of course, the TaubNUT soliton and boost are non-singular solutions too. The compactification of the Taub-NUT soliton yields the known KK-monopole in 4d, which has also only a compactification singularity.

Also on the heterotic side, we can find a solution that includes all four black hole types. This is the fundamental string lying inside a solitonic 5-brane, again with a boost along the fundamental string and a Taub-NUT soliton in transversal space. The metric is given by [15]

$$
\begin{array}{r}
d s_{h e t}^{2}=\frac{1}{H_{1}}\left(d u d v+H_{0} d u^{2}\right)+d z_{i}^{2}+ \\
+H_{2}\left(\frac{1}{H_{3}}\left(d x_{4}+\vec{V} d \vec{x}\right)^{2}+H_{3} d \vec{x}\right)
\end{array}
$$

$\left(\epsilon_{i j k} \partial_{j} V_{k}=\partial_{i} H_{3}\right)$. Strictly speaking it is not a genuine heterotic solution. Instead it is a solution of the NS-sector of all string theories. The reduction to $4 \mathrm{~d}$ can be done in two steps, first going to $6 \mathrm{~d}$ (compactifying $z_{i}$ ) and then to $4 \mathrm{~d}$. The internal space in the second step is determined by two scalar fields $T$ and $U$ and in addition we have the 4 d dilaton $S$. We write the internal metric as

$G_{r s}=\left(\begin{array}{cc}\frac{H_{0}}{H_{1}} & 0 \\ 0 & \frac{H_{2}}{H_{3}}\end{array}\right)=\frac{H_{2}}{H_{3}}\left(\begin{array}{cc}(\Im U)^{2} & 0 \\ 0 & 1\end{array}\right)$

and define the scalar fields by

$$
\begin{aligned}
& S=i e^{-2 \phi}=i e^{-2 \hat{\phi}} \sqrt{\left|G_{r s}\right|}=i \sqrt{\frac{H_{0} H_{1}}{H_{2} H_{3}}} \\
& T=i \sqrt{\left|G_{r s}\right|}=i \sqrt{\frac{H_{0} H_{2}}{H_{1} H_{3}}}, U=i \sqrt{\frac{H_{0} H_{3}}{H_{1} H_{2}}}
\end{aligned}
$$

( $\hat{\phi}$ is the $6 \mathrm{~d}$ dilaton). Note, that we are dealing with axion-free solutions, i.e. the real part of these fields vanish (see [16]).

What about supersymmetry? Generically every brane breaks $1 / 2$ of supersymmetry. But all supersymmetries are broken if the dimension of the relative transversal space is not 4 or 8 [17, 11] and they do not fulfil the equations of motion [10]. In combination with the requirement to have an asymptotically flat solution this restrict the maximal number of intersecting branes to four. If one relax the restriction of asymptotical flatness or if one is interested in compactifications to $3 \mathrm{~d}$ 
or $2 \mathrm{~d}$ one can have solutions with more than 4 branes [18]. We see, many branes break many supersymmetries. As consequence the renormalization behavior gets worse. For enough unbroken supersymmetries and small curvature the nonrenormalization theorems prevent the solutions to get quantum corrections. E.g. in $4 \mathrm{~d}$, solutions of $N=4,8$ supersymmetric theories are protected from quantum corrections but for solutions with only $N=2$ supersymmetry we have to expect quantum corrections on the one-loop and nonperturbative level. This is especially relevant for solutions with 3 or 4 intersecting branes. In the $\sigma$ model language on the heterotic side this means, that these background fields are zeros only of the zero-loop- $\bar{\beta}$-functions but not if we include the first genus. Also for solutions, where the curvature is not under control we have to expect $\alpha^{\prime}$ corrections. Even for an $N=4$ theory these terms are not protected by a non-renormalization theorem. In the next paragraph we will discuss the way of how one can address this question.

\section{Quantum aspects of $N=2$ black holes}

We are mainly interested in the compactified solution in 4 dimensions. All this solutions have a certain amount of supersymmetry and can be embedded into known supergravity theories, which give us a natural framework for the discussion of corrections. These are not only quantum correction. We have also corrections that are related to the topology of the internal space, e.g. in the case of CY compactifications. We will restrict ourselves on the $N=2$ case in which the symplectic geometry gives us a powerful tool.

Before we start we will fix our notations (see 19 and refs. therein). The $N=2$ supergravity includes one gravitational, $n_{v}$ vector and $n_{h}$ hyper multiplets. In what follows we will neglect the hyper multiplets, assuming that these fields are constant. The bosonic $N=2$ action is given by

$$
\begin{aligned}
S & \sim \int d^{4} x \sqrt{G}\left\{R-2 g_{A \bar{B}} \partial z^{A} \partial \bar{z}^{B}+\right. \\
& \left.+\frac{1}{4}\left(\Im \mathcal{N}_{I J} F^{I} \cdot F^{J}+\Re \mathcal{N}_{I J} F^{I} \cdot{ }^{\star} F^{J}\right)\right\}
\end{aligned}
$$

where the gauge field part $F^{I} \cdot F^{J} \equiv F_{\mu \nu}^{I} F^{J \mu \nu}$ and $I, J=0,1 \ldots n_{v}$. The complex scalar fields of the vector multiplets $z^{A}\left(A=1 . . n_{v}\right)$ parameterize a special Kähler manifold with the metric $g_{A \bar{B}}=\partial_{A} \partial_{\bar{B}} K(z, \bar{z})$, where $K(z, \bar{z})$ is the Kähler potential. Both, the gauge field coupling as well as the Kähler potential are given by the holomorphic prepotential $F(X)$

$$
\begin{aligned}
& e^{-K}=i\left(\bar{X}^{I} F_{I}-X^{I} \bar{F}_{I}\right) \\
& \mathcal{N}_{I J}=\bar{F}_{I J}+2 i \frac{\left(\Im F_{I L}\right)\left(\Im F_{M J}\right) X^{L} X^{M}}{\left(\Im F_{M N}\right) X^{M} X^{N}}
\end{aligned}
$$

with $F_{I}=\frac{\partial F(X)}{\partial X^{I}}$ and $F_{M N}=\frac{\partial^{2} F(X)}{\partial X^{M} \partial X^{N}}$ (these are not gauge field components). The scalar fields $z^{A}$ are defined by

$z^{A}=\frac{X^{A}}{X^{0}}$

and for the prepotential we take the general cubic form

$F(X)=\frac{1}{6} \frac{C_{A B C} X^{A} X^{B} X^{C}}{X^{0}}$

with general constant coefficients $C_{A B C}$. In type II compactification these are the classical intersection numbers of the Calabi Yau three fold. On the heterotic side these coefficients parameterize quantum corrections. The complete prepotential contains still further terms, e.g. we have omitted logarithmic and non-perturbative corrections. As long as we make sure that the scalars are large $\left(\left|z^{A}\right| \gg 1\right)$ this is a good approximation.

To find solutions to the above Lagrangian is in general very difficult. However, restricting on the Reissner-Nordstrøm case, i.e. with 4 non-trivial harmonic functions, there exists a special limit in which a solution can easily obtained. This is the double-extreme limit 20] where the scalar fields $z^{A}$ becomes constant everywhere. On the heterotic side these are the fields as defined in (14) $z^{1}=S, z^{2}=T$ and $z^{3}=U$ (if $n_{v}=3$ ). The values can be determined on the horizon where the central charge is extremal [21] and the symplectic coordinates have to fulfil the constraint (in a certain basis, see [19])

$X^{I}-\bar{X}^{I}=i p^{I}, \partial_{I} F(X)-\partial_{\bar{I}} F(\bar{X})=i q_{I}$

with $p^{I}$ and $q_{I}$ as the physical charges obtained by

$q_{I}=\int_{S_{\infty}} G_{I} \quad, \quad p^{I}=\int_{S_{\infty}} F^{I}$ 
from the gauge fields $F_{\mu \nu}^{I}$ and $G_{I \mu \nu}=\Re N_{I J} F_{\mu \nu}^{J}-$ $\Im N_{I J}{ }^{*} F_{\mu \nu}^{J}$. For the general cubic prepotential these constraints have been solved for the axionfree case in 19]. The solution with axions is not explicitely known - it is given in terms of an algebraic constraint 22. A special solution with non-constant scalar fields could be obtained by replacing the charges in (19) by harmonic functions [23]. This axion-free solution is

$$
\begin{aligned}
& d s^{2}=-e^{-2 U} d t^{2}+e^{2 U} d \vec{x} d \vec{x} \\
& e^{2 U}=\sqrt{H_{0} \frac{1}{6} C_{A B C} H^{A} H^{B} H^{C}}, \\
& z^{A}=i H_{0} H^{A} e^{-2 U}, \\
& F_{m n}^{A}=\epsilon_{m n p} \partial_{p} H^{A}, F_{00 m}=\partial_{m}\left(H_{0}\right)^{-1} .
\end{aligned}
$$

Note that $F_{I \mu \nu}=\mathcal{N}_{I J} F_{\mu \nu}^{J}$ and $\Im N_{I J}=\mathcal{N}_{I J}$, the real part vanishes. To be specific we choose for the harmonic functions

$H^{A}=\sqrt{2}\left(h^{A}+\frac{p^{A}}{r}\right), H_{0}=\sqrt{2}\left(h_{0}+\frac{q_{0}}{r}\right)$

where $h^{A}, h_{0}$ are constants which fixes the scalar fields at infinity. This solution looks like a "rotated version" of the solution with only 4 harmonic functions. We know from general duality invariant $N=4$ solutions that they have a similar structure (see e.g. the pure magnetic solution in [24). But already the special example discussed below tells us, that we cannot write this black hole solution in terms of only 4 harmonic functions with real coefficients.

The black hole carries one electric and $n_{v}$ magnetic charges related to the gauge fields $F_{0 m}^{0}$ resp. $F_{m n}^{A}$. To get the mass we have to look on the asymptotic geometry. First, in order to have an asymptotically flat Minkowski space we have the constraint

$4 h_{0} \frac{1}{6} C_{A B C} h^{A} h^{B} h^{C}=1$.

Then, we get asymptotically

$e^{-2 U}=1-\frac{2 M}{r} \pm \ldots$

and the mass is

$M=\frac{q_{0}}{4 h_{0}}+\frac{1}{2} p^{A} h_{0} C_{A B C} h^{B} h^{C}$.
As expected our solution saturates the BPS bound

$M^{2}=|Z|_{\infty}^{2}=\left(q_{0} X^{0}-p^{A} F_{A}\right)_{\infty}^{2}$

where the r.h.s. has to be calculated at spatial infinity $\left(e_{\infty}^{U}=1\right)$.

On the other side if we approach the horizon all constants $h^{A}$ and $h_{0}$ drop out. The area of the horizon depends only on the conserved charges $q_{0}, p^{A}$. Furthermore, if $q_{0} C_{A B C} p^{A} p^{B} p^{C}>0$ the solution behaves smooth on the horizon and we find for the area and entropy $(\mathcal{S})$

$A=4 \mathcal{S}=4 \pi \sqrt{4 q_{0} \frac{1}{6} C_{A B C} p^{A} p^{B} p^{C}}$.

If the charges and $h^{\prime} s$ are positive the area of the horizon defines a lower bound for the mass. Minimizing the mass gives us the area of the horizon 21]

$\left.4 \pi M^{2}\right|_{\text {min. }}=A$.

In this case all scalar fields are constant and

$h_{0}=\frac{q_{0}}{c} \quad, \quad h^{A}=\frac{p^{A}}{c}$

where $c^{4}=4 q_{0} \frac{1}{6} C_{A B C} p^{A} p^{B} p^{C}$. In order to verify this, one has first to replace $h_{0}$ in (25) by using the constraints (23). Then, using (29) as ansatz for $h^{A}$ one finds that: $\partial / \partial h^{A} M=0$ for all $A$ and thus, these values extremize the mass. On the other side if some of the $p^{A}$ are negative, we can have massless black holes and obviously (27) cannot be the minimum.

For these moduli all scalars are constant, i.e. coincide with their value on the horizon $\left(z^{A} \equiv\right.$ $\left.\left.z^{A}\right|_{h o r}.\right)$. By this procedure we get the double extreme black hole [20] and taking this limit, our solution (21) coincides with the solution given in [19]. There is yet another way to look on this extremization. The moduli fields are dynamical fields in $N=2$ supersymmetric gauge theories. Especially, the values at infinity $\left(h^{A}, h_{0}\right)$ are not protected by a gauge symmetry. For a given model there is no way to fix these values. Instead one could argue that the model chooses those values for which the energy or ADM mass is minimal, i.e. the double extreme case. This is 
the notion of dynamical relaxation that has been introduced in [25].

Let us end this paragraph with a discussion of the quantum corrections on the heterotic side. To be explicit we consider the 3 -moduli model with the prepotential

$$
F(X)=\frac{X^{1} X^{2} X^{3}}{X^{0}}+\frac{1}{3} \frac{\left(X^{3}\right)^{3}}{X^{0}}
$$

where the first term corresponds to the classical $S T U$ model as described in paragraph 3 and the second is a quantum correction [26]. For this model the metric is $\left(p^{A} \rightarrow \frac{p^{A}}{\sqrt{2}}, q_{0} \rightarrow \frac{q_{0}}{\sqrt{2}}\right)$

$$
\begin{aligned}
& d s^{2}=-e^{-2 U} d t^{2}+e^{2 U} d \vec{x}^{2} \\
& e^{4 U}=H_{0} \frac{1}{6} C_{A B C} H^{A} H^{B} H^{C}=\left(1+\frac{q_{0}}{r}\right) . \\
& \cdot\left(1+\frac{p^{3}}{r}\right)\left(\left(1+\frac{q^{1}}{r}\right)\left(1+\frac{p^{2}}{r}\right)+\frac{1}{3}\left(1+\frac{p^{3}}{r}\right)^{2}\right) .
\end{aligned}
$$

We see that the quantum term acts as a regulator. E.g. if $q^{1}, p^{2}<0$ the singularity at $r=-q^{1}, p^{2}$ is regularized, which is the massless black hole singularity. The solution behaves smooth even for vanishing $q^{1}$ and $p^{2}$, which is classically not the case. But these questions deserves further investigations, since near these points the validity of this simple solution is questionable.

The model gives a realistic picture as long as $H_{0} \gg H^{1}>H^{2}>H^{3} \gg 1$. The first inequality ensures that $S, T$ and $U$ are large and all further corrections in the prepotential are sufficiently suppressed. The last inequality suppressed higher curvature corrections ( $\alpha^{\prime}$ corrections), since the radius of the throat in the stringframe is proportional to the magnetic charges (see figure 1). Near the horizon, the above inequalities can directly be translated into relations between the charges. If we have however negative charges, the harmonic functions have zeros and near these points our approximation breaks down.

For the mass and entropy we get also additive corrections

$$
\begin{aligned}
& M=M_{c l}+\frac{1}{4} p^{3}, \\
& \mathcal{S}=2 \pi \sqrt{q_{0} q^{1} p^{2} p^{3}+\frac{1}{3} q_{0}\left(p^{3}\right)^{3}} .
\end{aligned}
$$

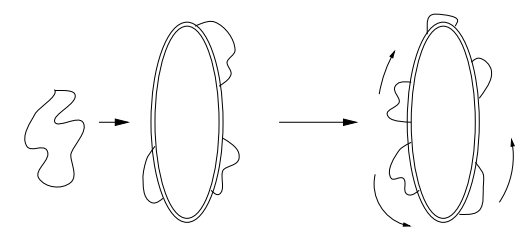

Figure 4. All open strings are moving in one direction along the closed string built out of multiple layers of $D$-branes. If another closed string falls in, it splits in two pieces one moving right the other moving left.

\section{Microscopic entropy picture}

From the thermodynamics we know that the entropy should count the degeneracy of states that build a physical system. For long time it has been an open question what is this meaning for the Bekenstein-Hawking entropy of black holes. What states do we have to count and where are they located? In the last year a complete new picture of black holes emerged, the $D$-brane picture [27], [28]. The main idea is that the black holes are wrapped $D$-branes (or $M$-branes) with open string (or membrane) states on their surface. If we are far away it looks like the standard black hole, but if we approach it, we see that the surface is not smooth, but consists of clouds of open strings attached to the horizon (let us assume that the horizon is non-singular). At the same time we will see that the black hole (21) is not really a point, but it is a string like object. This is at least the case if we assume that the electric charge is large compared to the magnetic ones, which in turn is a consistency relation to keep further corrections under control. If the black hole is extremal (BPS solution) the open strings move in one direction only.

In this picture the excitation and the following evaporation of the black hole has also a nice description (see figure 4 and 5). Taking a closed string and throwing it into the black hole, it will break up into two open strings, one will be right- and the other left-moving. The black hole is excited or non-extremal. The part, which 


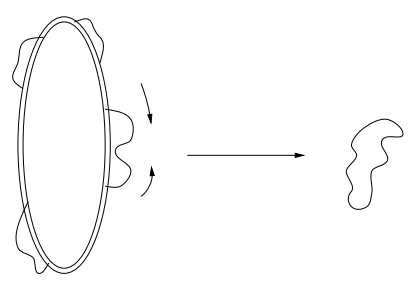

Figure 5. Two open strings with opposite momenta can again recombine and build a closed string. This is for $D$ branes the qualitative picture of the black hole evaporation.

moves in the opposite direction to all other open strings, will eventually recombine with another open string and build a closed string that can escape to infinity. In this picture seems to be no place for an information loss, nothing is lost every state "moves on the horizon".

A question remains however: why can the closed string not move behind the horizon and is trapped there? In answering this question one could argue that the discussion so far was a little bit naive. Let us repeat here some arguments given in [29]. To talk about states makes sense only in regions where the coupling to gravity is sufficiently small, i.e. where we can regard the (open string) states as small fluctuation around flat space time. If the coupling increases a single state has no sense. The back-reaction of gravity and all other fields will mix up all states. If the coupling is large enough the complete system will collapse into a black hole (or black string). A flat space description exists if the gravitational field strength vanish for vanishing coupling. From scaling arguments it follows that this is only the case for electric NS solutions and the RR solutions, but not for magnetic NS solutions. Since our black holes are dyonic only the RR charged solution has a flat space description at weak coupling: in terms of $D$-branes and open strings. Coming back to the question above, we can now argue that the described $D$-brane picture just appears in the weak coupling regime. There, we do not have a horizon, we have branes in flat space time and open strings moving freely on these branes. Increasing now the gravitational coupling the system collapse and our black hole (or black string) is created. In the hope that the transition between both regions is smooth, we can qualitatively describe the system in the $D$-brane picture. I.e. we can give an explanations of the Hawking radiation and also can count the states relevant for the Bekenstein-Hawking entropy.

Let us now come to the question of state counting for our solution. This has been done already for other solutions, which correspond to $K 3$ compactification [27] and the torus case [28]. We will concentrate here on the modifications that appear for the CY-compactification.

Our solution can be obtained when we take 3 intersecting $M$-5-brane and wrap them in the internal space. In the case that the internal space is a torus this configuration is given in eq. (10). On the heterotic side this corresponds to the classical case. For the state counting it is useful first to compactify the $6 \mathrm{~d} \omega^{A}$ part, which gives us a string solution in $5 \mathrm{~d}$. Wrapping this string around a further circle yields our black hole in $4 \mathrm{~d}$. The states of the black hole can then be identified with the states of the string in $5 \mathrm{~d}$. For the extremal case this state counting has been done in [30] (see also [31] for the CY case of the type II theory). Let us first repeat the arguments for the extremal case and later discuss the non-extremal generalization.

The states that we have to count are open membranes attached to the $M$-5-branes [12]. After compactification, the open membranes become open string states and since we want to consider first the extremal case (BPS limit), they all travel in one direction. For the $5 \mathrm{~d}$ string these states are equivalent to momentum modes which yield the electric charge of our solution (21) upon compactification to 4 dimensions. Since the $M$ 5-brane can be wrapped many times around the CY-cycles, the $5 \mathrm{~d}$ string consist of many layers to which the states can be attached. This picture is shown in figure 6 .

For the torus compactification a counting procedure has been proposed in [32] and we will adopt their arguments here. Before we start to count let us describe the states shortly. The open membranes in 11 dimensions connect all three 5branes. As consequence the open string states 


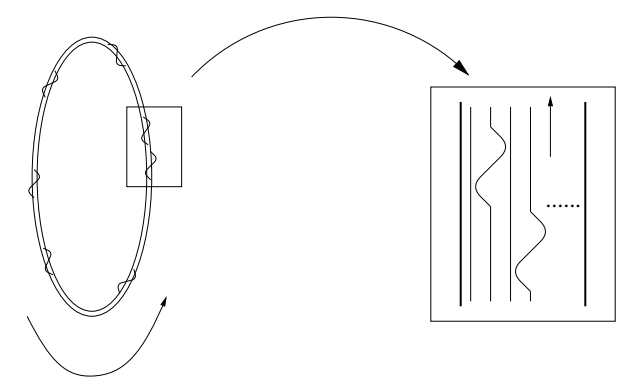

Figure 6. This figure shows the 5-dimensional string with open string states (=momentum modes) travelling in one direction (BPS limit). In the simplest case the string consist of $p^{1} p^{2} p^{3}$ layers

in $5 \mathrm{~d}$ consist of three open strings attached to the boundary at three points. They scale like $p^{3}$. What about other states? Membranes with less than 3 boundaries are in general there, but they are subleading for large charges (scale like $p^{2}$ ). On the other side membranes with more than 3 boundaries would destroy our picture, since the Bekenstein-Hawking entropy scales like $p^{3}$. One can however argue that these membranes have to have at least two boundaries on one 5-brane. On the other side, every stretched open membrane becomes massive and it tries to get the least mass, i.e. either the two boundaries will recombine or they will move to a self-intersection of the cycle and the state becomes massless there. For a torus there are no self-intersections and thus only the open membranes with 3 boundaries are stable, branes with more boundaries will join all boundaries at the same brane. However, in the CY case we do have self-intersections and we do have stable (massless) open membrane states with more boundaries on the same 5-brane. But in every CY-3-fold at most three 4-cycles (different of the same one) can intersect in one point and thus only open membranes with 3 boundaries can be stable and have the right scaling behavior $\left(\sim p^{3}\right)$. So, for our situation only the open membrane with 3 boundaries contribute to the statistical entropy.
Next, how can we be sure that every state appears on the $5 \mathrm{~d}$ string, that nothing is hidden in the internal space? In wrapping the branes around the internal cycles, we can shift the layers a little (see figure 7). Or equivalently one could consider parallel (not coinciding) 5-branes. As consequence, the open membranes are stretched at any point in the internal space and they will move to the intersections, to become massless there. The special case, that the 5-branes coincide in the internal space does not change the number of states, only the location.

Now we can start to count these states and compare the Bekenstein-Hawking entropy with the statistical entropy. For a large left-moving oscillator number $N_{L}=q_{0}$ the statistical entropy of a string is given by

$S_{\text {stat. }}=\log d\left(N_{L}\right)=2 \pi \sqrt{\frac{1}{6} c_{e f f} N_{L}}$

where $c_{\text {eff }}$ is the effective central charge which is given to leading order by $c_{e f f}=D_{e f f}\left(1+\frac{1}{2}\right)$ (bosonic plus fermionic modes). Here, $D_{\text {eff }}$ is the effective number (= dimension) of oscillating modes and it is given by the number of layers times the number of oscillating modes for every layer. The number of layers is given by the number of how many times we wrap a branes around the internal cycles. Lets assume that we have three 4-cycles and wrap the first brane $p^{1}$ times, the second brane $p^{2}$ and the third brane $p^{3}$ times. Note, that we assume that the 5-branes have a proper normalized charge in 10 dimensions. The charges in $4 \mathrm{~d}$ comes from multiple wrapping of the 5 -brane. At the common intersection we have $p^{1} p^{2} p^{3}$ branes lying on top of each other (see figure 7). Since each of these layers is part of one $M$-5-brane the oscillating modes are oscillations of the 5-brane, which are the 4 transversal oscillations. As result we find that $D_{\text {eff }}=4 \cdot p^{1} p^{2} p^{3}$. This discussion can immediately generalized to the case that we have $n_{v}$ 4-cycles around which we can wrap 5-branes. In this case we assume that we have wrapped a 5 -brane $p^{A}$ times around the Ath 4-cycle $\left(A=1, . ., n_{v}\right)$. Assuming for the moment that we do not have self-intersections, this can be written as $\frac{1}{6} C_{A B C} p^{A} p^{B} p^{C}$, using that $C_{A B C}$ is symmetric and counts the number of intersections of the 4 -cycles. The resulting entropy 


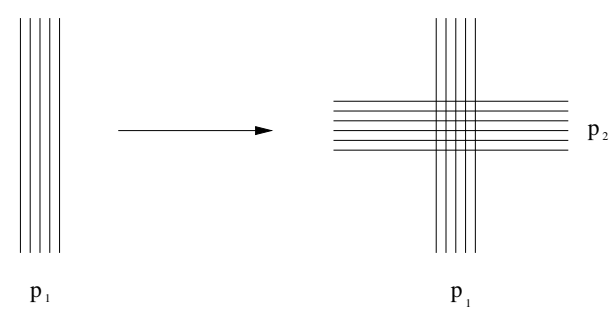

Figure 7. Wrapping a brane $p^{1}$ times around a 4 -cycle gives $p^{1}$ branes lying on top of each other. Intersecting two such 4 -cycles yield $p^{1} p^{2}$ branes that lying on the common intersection.

is

$S_{\text {stat }}=2 \pi \sqrt{q_{0} \frac{1}{6} C_{A B C} p^{A} p^{B} p^{C}}$

and coincides with the Bekenstein-Hawking entropy (27) and for the 3-moduli case with the entropy found in 33.

What about self-intersections? At the beginning of our discussion we had argued that the open membranes can sit with their boundaries on the same 5-brane, but only at self-intersections. In this case the endpoints are not distinguishable and we have to divide out the permutation of the endpoints sitting on the same brane, i.e. we have the combinatoric factor $\frac{1}{6}=\frac{1}{3 !}$ if all boundaries are on the same brane. Hence, the statistical entropy (34) includes also the cases of self-intersections.

Finally, let us come to the non-extremal case. In paragraph 2 we have seen how we can make our solution non-extremal. For the entropy counting this means that we also have to include rightmoving modes. Let us explain this. In order to make a brane intersection non-extremal we have to introduce for every harmonic function a boost parameter (see second ref. of [4]). The appearance of these parameters can be understood as follows. One can start with the Schwarzschild black hole and boost it with finite velocity in one direction. Next, we $T$-dualize this direction and get a non-extremal fundamental string. By $S$-duality we convert it to a $D$-string and finally by further
$T$-duality we can create all other non-extreme $D$ branes. In order to get an intersection we take, e.g., a non-extreme $N S$-5-brane. Again we boost this brane along one of their world-volume coordinates and then by $S$ - and $T$-duality we can obtain all non-extreme intersections. This procedure can be continued. So, for every brane we get a different boost parameter.

This procedure gives the right fields for toroidal compactifications and the 4-d metric reads, see e.g. 伍

$$
\begin{aligned}
d s^{2} & =-e^{-2 U} f d t^{2}+e^{2 U}\left(\frac{d r^{2}}{f}+r^{2} d \Omega\right), \\
e^{2 U} & =\sqrt{\tilde{H}_{0} \tilde{H}^{1} \tilde{H}^{2} \tilde{H}^{3}},
\end{aligned}
$$

where: $f=1-\frac{\mu}{r}, \tilde{H}^{A}=\sqrt{2}\left(h^{A}+\frac{\tilde{p}^{A}}{r}\right)$ and $\tilde{p}^{A}=\mu \sinh ^{2} \alpha^{A}$. Now, the horizon is not $r=0$ but at $r=\mu$. We have to keep in mind that this solution is not supersymmetric. This means that it gets many more corrections. We do not have anymore the tool of special geometry, which we used in paragraph 4 . But as long as we keep $\mu$ very small compared to the charges and the boost parameter large, we can see this solution as a small deviation from the extremal solution. In the $D$-brane picture this means we have thrown only very few states into a fat black hole.

The Hawking temperature can be obtained by going to the Euclidean time. In order to avoid a conical singularity on the horizon, this Euclidean time has to be periodic and as usual the periodicity determines the temperature

$0 \leq t_{e} \leq \beta=\frac{1}{T_{H}}$.

Thus, we find for the Hawking temperature and the entropy, which is again proportional to the area of the horizon (setting $h^{A}=h_{0}=1$ )

$$
\begin{aligned}
& S=2 \pi \sqrt{\hat{q}_{0} \hat{p}^{1} \hat{p}^{2} \hat{p}^{3}} \\
& T_{H}=\frac{\mu}{4 \pi \sqrt{\hat{q}_{0} \hat{p}^{1} \hat{p}^{2} \hat{p}^{3}}} .
\end{aligned}
$$

with: $\hat{p}^{A}=\tilde{p}^{A}+\mu=\mu \cosh ^{2} \alpha^{A}$. The entropy has the same structure as before, only that it is expressed now in term $\hat{p}$. The extreme limit is given by $\mu \rightarrow 0$ and $p=\tilde{p}=\hat{p}$. In this case the Hawking temperature vanish, or equivalently the Euclidean time becomes non-compact. 
Following the procedure of 34, we can also give this entropy a statistical interpretation. The main idea is, that the energy of the in-following states is used to excite the wrapped branes, brane - antibrane pairs are created. At the same time the in-falling state (closed string) splits up into two pieces, one is left-moving and the other rightmoving (see figure 4). In order to motivate this idea one looks on the physical charges, which are given by the non-extreme field strength. In the non-extreme case they become

$$
\begin{aligned}
q_{0} \rightarrow & \mu \cosh \alpha_{0} \sinh \alpha_{0}= \\
& =\frac{\mu}{4}\left(e^{2 \alpha_{0}}-e^{-2 \alpha_{0}}\right)=q_{0}-\bar{q}_{0}, \\
p^{A} \rightarrow & \mu \cosh \alpha^{A} \sinh \alpha^{A}= \\
& =\frac{\mu}{4}\left(e^{2 \alpha^{A}}-e^{-2 \alpha^{A}}\right)=p^{A}-\bar{p}^{A}
\end{aligned}
$$

if: $q_{0}$ and $p^{A}$ are our extreme charges and $\bar{q}_{0}$ and $\bar{p}^{A}$ are charges of anti-branes. Both numbers have to be integer and counts the number branes and antibranes, or the left- and right-moving momentum modes $\left(q_{0}=N_{L}\right.$ and $\left.\bar{q}_{0}=N_{R}\right)$. Adopting the notation of [34] we can replace in (37)

$$
\begin{aligned}
& \sqrt{\hat{q}}=\sqrt{N_{L}}+\sqrt{N_{R}}, \\
& \sqrt{\hat{p}^{A}}=\sqrt{N_{5}^{A}}+\sqrt{N_{\overline{5}}^{A}}
\end{aligned}
$$

with: $N_{5}^{A}=p^{A}, N_{\overline{5}}^{A}=\bar{p}^{A}$. So, in addition to momentum modes travelling on the original branes we have now also to sum over oscillations of the anti-brane part as well as right-moving modes.

Note, that the brane-antibrane picture is only very qualitative. It is not a bound state, where we can separate only the anti-brane part. For a vanishing brane part the antibrane part vanishes too. Thus, it is on completely different footing as the non-threshold bound states of branes discussed in recent literature (see e.g. [35]).

\section{Conclusion}

We have reviewed some recent developments in $4 \mathrm{~d}$ black hole solutions, that can be embedded in $N=2$ supersymmetric theories. In comparison to $N=4$ solutions we had taken into account on the heterotic side quantum correction and on the type II side correction, that come from the CY compactification. Our model is described by a general cubic prepotential and has one electric and $n_{v}$ magnetic charges.

The $4 \mathrm{~d}$ solution can be seen as a compactification of three $M$-5-branes. If one first compactify over the CY-3-fold we get in $5 \mathrm{~d}$ a string solution, which is the common world-volume of all $M-5$ branes. The number of wrapping one 5-brane around a 4-cycles gives us the magnetic charge that corresponds to this 4-cycle. So the $5 \mathrm{~d}$ string consist of many branes lying on top of each other and along all of these layers we have momentum modes travelling around the string. This gives us a microscopic picture of the black hole and by counting of all states we were able to determine the degeneracy to a given charge configuration, which coincides with the statistical as well as Bekenstein-Hawking entropy. Making the black hole non-extremal means, that the branes get excited, they split into a brane and antibrane part and for the momentum modes we have left as well as right moving modes. This microscopic picture has been described in the last paragraph.

Still an open question is the inclusion of instanton corrections on the type II side. These terms correspond to logarithmic corrections in the prepotential. Especially in order to describe the massless states these corrections are important. In our approach we could suppress these terms by assuming that all charges are large, i.e. we considered a large black hole.

\section{Acknowledgements}

I would like to thank Thomas Mohaupt for numerous discussions. Work is supported by the DFG. 


\section{REFERENCES}

1. J. Dai, R.G. Leigh and J. Polchinski, Mod. Phys. Lett. A4(1989)2073.

2. J. Polchinski, Phys. Rev. Lett. 75(1995)4724, hep-th/9510017.

3. G.W. Gibbons and K. Maeda, Nucl. Phys. B298(1988) 741.

4. M.J. Duff, H. Lü and C.N. Pope, Phys. Lett. B382(1996)73, hep-th/9604052; M. Cvetič and A.A. Tseytlin, Nucl. Phys. B478(1996)181, hep-th/9606033.

5. J. Rahmfeld, Phys. Lett. B372(1996)198, hep-th/9512089.

6. E. Bergshoeff, M. de Roo and S. Panda, hepth/9609056.

7. R. Güven, Phys. Lett. B276(1992)49.

8. G. Papadopoulos and P. Townsend, Phys. Lett. B380(1996)273, hep-th/9603087.

9. A.A. Tseytlin, Nucl. Phys. B475(1996)149, hep-th/9604035.

10. K. Behrndt, E. Bergshoeff and B. Jansen, hep-th/9604168.

11. V. Balasubramanian and F. Larsen, hepth/9604189; J.P. Gauntlett, D.A. Kastor and J. Traschen, Nucl. Phys. B478(1996)544, hep-th/9604179.

12. A. Strominger, Phys. Lett. B383(1996)44, hep-th/9512059; P. Townsend, Phys. Lett. B373(1996)68, hep-th/9512062.

13. G.W. Gibbons, G.T. Horowitz and P.K. Townsend, Class.Quant.Grav 12(1995)297, hep-th/9410073.

14. K. Behrndt and E. Bergshoeff, Phys. Lett. B383(1996)383, hep-th/9605216.

15. M. Cvetič and A.A. Tseytlin, PRD53(1996)5619, hep-th/9512031.

16. M.J. Duff, J.T. Liu, J. Rahmfeld, Nucl. Phys. B459(1996)125, hep-th/9508094.

17. J. Polchinski, S. Chaudhuri and C.V. Johnson, hep-th/9602052.

18. E. Bergshoeff, M. de Roo, E. Eyras, B. Janssen and J.P. van der Schaar, hepth/9612095.

19. K. Behrndt, G. Lopes Cardoso, B. de Witt, R. Kallosh, D. Lüst and T. Mohaupt, hepth/9610105.

20. R. Kallosh, M. Shmakova and W.K. Wong, Phys. Rev. D54(1996)6284, hep-th/9607077.
21. S. Ferrara, R. Kallosh and A. Strominger, Phys. Rev. D52(1995)5412, hep-th/9508072;

S. Ferrara and R. Kallosh, hep-th/9602136.

22. M. Shmakova, hep-th/9612076.

23. K. Behrndt, hep-th/9610232.

24. K. Behrndt and R. Kallosh, Phys. Rev. D53(1996)589, hep-th/9509102.

25. S.-J. Rey, hep-th/9610157.

26. J.A. Harvey and G. Moore, Nucl. Phys. B463(1996)315, hep-th/9510182.

27. A. Strominger and C. Vafa, Phys. Lett. B379(1996)99, hep-th/9601029; R. Dijkgraaf, E. Verlinde and H. Verlinde, hepth/9607026.

28. C.G. Callan and J.M. Maldacena, hepth/9602043; J.M. Maldacena and A. Strominger, Phys. Rev. Lett. 77(1996)428, hepth/9603060.

29. G. Horowitz, gr-qc/9604051.

30. K. Behrndt and T. Mohaupt, hep-th/9611140.

31. J. Maldacena, hep-th/9611163.

32. I. Klebanov and A.A. Tseytlin, Nucl. Phys. B475(1996)179, hep-th/9604166.

33. K. Behrndt, R. Kallosh, J. Rahmfeld, M. Shmakova, W.K. Wong, Phys. Rev. D54(1996)6293, hep-th/9608059; G. Lopes Cardoso, D. Lüst and T. Mohaupt, hepth/9608099.

34. G.T. Horowitz and A. Strominger, Phys. Rev. Lett. 77(1996)2368, hep-th/9602051; G.T. Horowitz, J.M. Maldacena and A. Strominger, Phys. Lett. B383(1996)151, hepth/9603109.

35. J.G. Russo and A.A. Tseytlin, hep-th/9611047. 